\title{
Evidence of a Media-Induced Nocebo Response Following a Nationwide Antidepressant Drug Switch
}

\author{
Kate MacKrill ${ }^{\mathrm{a}}$, Greg D. Gamble ${ }^{\mathrm{b}}$, Debbie J. Bean ${ }^{\mathrm{a}}$, Tim Cundy ${ }^{\mathrm{b}}$, Keith J. Petrie ${ }^{\mathrm{a}}$ \\ [a] Department of Psychological Medicine, University of Auckland, Auckland, New Zealand. [b] Department of Medicine, \\ University of Auckland, Auckland, New Zealand.
}

Clinical Psychology in Europe, 2019, Vol. 1(1), Article e29642, https://doi.org/10.32872/cpe.v1i1.29642

Received: 2018-09-10 • Accepted: 2018-12-05 • Published (VoR): 2019-03-29

Handling Editor: Winfried Rief, Division of Clinical Psychology and Psychotherapy, Department of Psychology, Philipps-University of Marburg, Marburg, Germany

Corresponding Author: Keith J. Petrie, Psychological Medicine, Faculty of Medical and Health Sciences, University of Auckland, Private Bag 92019, Auckland, New Zealand. E-mail: kj.petrie@auckland.ac.nz

\section{Abstract}

Background: In 2017, patients on a generic or branded antidepressant venlafaxine were switched to a new generic formulation (Enlafax). In February and April 2018, two major NZ media outlets ran stories about the new generic being less effective and causing specific side effects. This study aimed to examine the effect of the media coverage on drug side effects reported to the national Centre for Adverse Reactions Monitoring (CARM) and whether the specific symptoms reported in the media increased compared to side effects not reported in the media.

Method: We analysed monthly adverse reaction reports for Enlafax to CARM from October 2017 to June 2018 and compared adverse reports, complaints of decreased therapeutic effect and specific symptom reports before and after the media coverage using an interrupted time series analysis.

Results: We found the number of side effects and complaints of reduced therapeutic effect increased significantly following the media stories (interruption effect $=41.83,95 \%$ CI $[25.25$, $58.41], \mathrm{p}=.003$; interruption effect $=15.49,95 \% \mathrm{CI}[7.01,23.98], \mathrm{p}=.012$, respectively). The specific side effects mentioned in the media coverage, including suicidal thoughts, also increased significantly compared to other side effects not mentioned in the media.

Conclusions: In the context of a drug switch, media reports of side effects appear to cause a strong nocebo response by increasing both the overall rate of side effect reporting and an increase in the specific side effects mentioned in the media coverage, including reduced drug efficacy and heightened suicidal thoughts.

\section{Keywords}

media, nocebo effect, venlafaxine, side effects, generic medicines 


\section{Highlights}

- The study provides further evidence that media coverage of side effects can induce a nocebo effect.

- This is the first study to look at media coverage of an antidepressant brand switch.

- The increase in reported adverse events was higher for those symptoms mentioned in the media reports.

Switches from branded to generic medicine formulations have become more frequent in recent times as health funders seek to reduce costs. These switches to generic medical and psychotropic medications have from time to time caused an increase in reported adverse events (Desmarais, Beauclair, \& Margolese, 2011; Leclerc et al., 2017), and this is likely to be due to negative attitudes towards generic medicines rather than pharmacological differences between the branded and generic versions of the medication (Colgan et al., 2015).

This phenomenon is known as the nocebo effect and research using inert medicines has shown that people report a reduced therapeutic effect and more side effects from a generic-labelled placebo compared to a branded placebo (Faasse, Martin, Grey, Gamble, \& Petrie, 2016). Similarly, the process of switching from one placebo tablet to another is associated with reports of side effects and reduced drug efficacy (Faasse, Cundy, Gamble, \& Petrie, 2013). The nocebo effect can also occur in active medications and there is recent evidence that media coverage about drug side effects can create a nocebo response by highlighting negative reactions to a particular medication and prompting an increase in symptom complaints and drug discontinuation (Faasse \& Petrie, 2013).

In 2017, 45,000 New Zealand patients prescribed the antidepressant venlafaxine were switched to a new funded generic (Enlafax XR) from either the branded originator or a different generic version. In February 2018, two major print and online media outlets in New Zealand ran stories on patients' complaints that the new generic was less effective and causing an increase in various symptoms, including heightened suicidal thoughts. A few months later, another media report was released, again discussing patients' reports of ineffectiveness and side effects from Enlafax. Based on previous research, we tested two hypotheses: firstly, that media coverage of the complaints following the venlafaxine switch would be associated with an increase in adverse drug reactions reported to the New Zealand Centre for Adverse Reactions Monitoring (CARM); and secondly, that the specific side effects reported in the media would increase compared to other side effects not reported in the coverage. 


\section{Method}

\section{Newspaper Articles}

On February 28 2018, two leading New Zealand media outlets, The New Zealand Herald (NZME) and Stuff (Fairfax Media), published newspaper and online articles on the venlafaxine brand switch. The New Zealand Herald ran a story titled "Patients say generic Pharmac-funded version of antidepressant venlafaxine left them depressed, anxious" (Henry, 2018), while Stuff's article was titled "Anti-depressant swap: Sufferers claim generic drug is harming their condition" (Maude, 2018). These articles described the personal experience of two patients when they switched from their original brand Efexor to Enlafax. The reports stated that the new, cheaper generic version was not as efficacious in managing the patients' depression and they were also experiencing side effects. The New Zealand Herald article specifically mentioned that patients were reporting suicidal thoughts, nausea, fatigue, headaches and anxiety.

In April, Stuff released another online article, which continued on the subject of the previous media coverage. This media report, "Fight over Pharmac's switch to generic anti-depressant brand continues" (Steele, 2018), again stated that the new generic was not as effective and noted that various adverse events had been reported - specifically headaches, anxiety and suicidal thoughts. Of the two websites, Stuff is the most viewed with approximately 161,600 unique views per day, while The New Zealand Herald has 94,800 views (https://www.siteprice.org). Neither of these stories suggested patients report side effects to their doctor or to CARM.

\section{Adverse Drug Reactions}

A report of all adverse reactions to venlafaxine was obtained from CARM through Medsafe, New Zealand's medicines monitoring agency. The CARM database collects adverse reactions to medicines and vaccines, the majority of which are submitted by healthcare professionals. Reporting is usually made online and CARM reporting forms can be accessed on the website https://nzphvc.otago.ac.nz. Reports were obtained from October 2017 to July 2018 and included the month the report was received, the patients' age and gender, and up to five symptoms attributed to the medicine. As the data was anonymous and publicly available, the study did not require ethical approval.

\section{Measures}

The number of reports of decreased therapeutic response was calculated for each month. Decreased therapeutic response is an adverse reaction category on the CARM database equivalent to a reduced efficacy of the medicine. 
The total number of side effects reported each month was also calculated as was the number of times the five specific side effects mentioned in the New Zealand Herald article were reported. The side effects were matched to the corresponding adverse reactions in the CARM database with headaches, nausea and anxiety being matched exactly. Two of the media-mentioned side effects were considered broad enough to cover a range of CARM adverse reactions. As such, reports of fatigue, lethargy and tiredness were grouped under the broader side effect of fatigue, while suicidal thoughts were matched with reports of suicidal tendencies, suicidal ideation and impulses to self-harm. The five most common adverse reactions not mentioned in the media reports were identified from the CARM database and used as control symptoms. These were dizziness, drug withdrawal syndrome, irritability, sleep disturbance and a fuzzy head.

\section{Statistical Analyses}

Interrupted time series analyses were conducted to investigate whether the February and April media reports on the venlafaxine brand switch were associated with an increase in the CARM reporting of decreased therapeutic response, total number of side effects and the specifically mentioned side effects in the months directly after the media reports compared to the five months before. An autoregressive integrated moving average (ARIMA) model with an autoregression term of 1 and moving average term of 1 was used for all analyses. As the three media reports were a one-off interruption to the normal time series, a binary independent variable was created to indicate their presence by month. March and May were given the value of 1 as these were the periods directly after the February and April media reports and all other months were coded 0 . The analysis produces an estimated interruption effect, which is the change in the rate of adverse event reporting from the months coded 0 and 1 , and indicates whether this change is significantly different. This is a more conservative analysis as the adverse event reports in March and May are averaged together to calculate the general effect of the three media stories rather than both months being compared separately to the pre-media rate. Analyses were conducted in SAS (v9.4 SAS Institute Inc., Cary, NC) using the SAS PROC ARIMA procedure. An alpha level of .05 was considered significant.

\section{Results}

In total, there were 100 adverse event reports from October 2017 to July 2018. The average age of reporters was 43.7 years old and $70.0 \%$ were female. In the five months prior to the first newspaper articles, the average number of adverse event reports to CARM was $6.00(S D=1.23)$ per month. In March and May, the two months directly after the release of the articles, the average number of CARM reports significantly increased to 25.50 
$(S D=12.02$; interruption effect i.e. difference between the pre-media average and March + May average $=19.45,95 \%$ confidence interval $(\mathrm{CI})[10.77,28.13], p=.005)$.

The newspaper articles also had a significant effect on side effect reporting with the pre-media average of 7.00 reports $(S D=4.18)$ a month increasing to $49.00(S D=26.63)$ in March and May, see Figure 1. Similarly, the rate of decreased therapeutic response reporting significantly increased from $4.00(S D=2.12)$ during the previous months to an average of $17.00(S D=9.90)$ over March and May. The interruption effect of the media on side effect reporting $=41.83,95 \%$ CI $[25.25,58.41], p=.003$. Interruption effect for decreased therapeutic response reports $=15.49,95 \%$ CI [7.01, 23.98], $p=.012$.
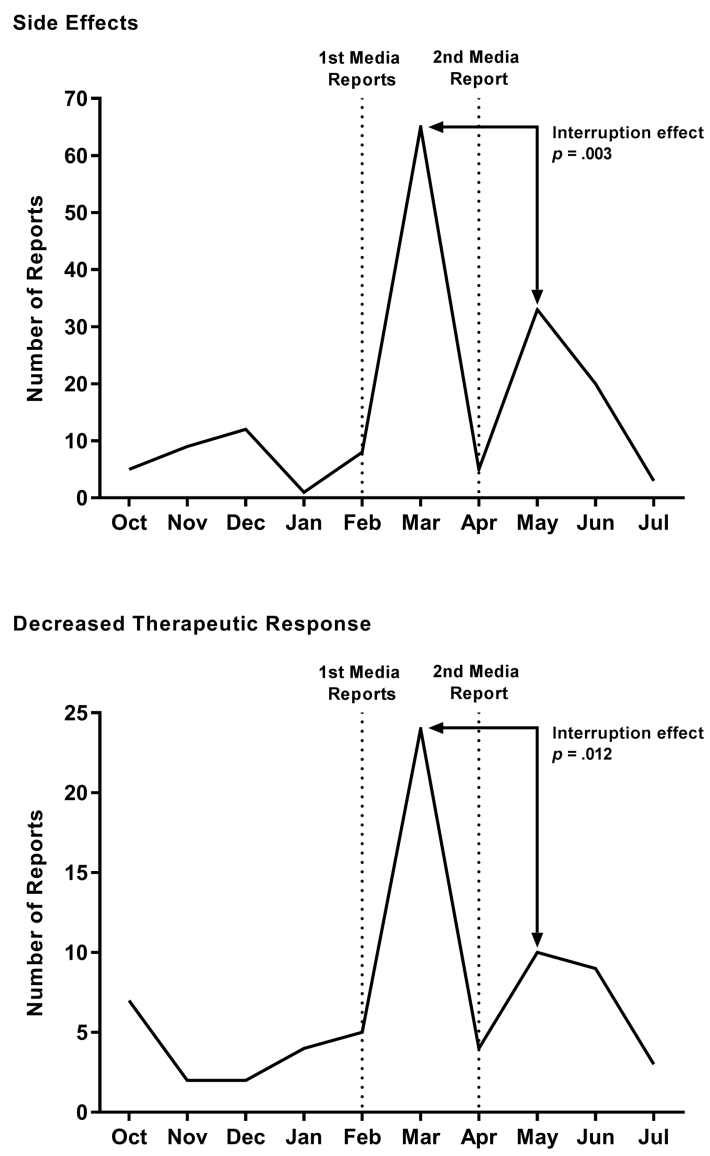

Figure 1. Number of reports of side effects and decreased therapeutic response before and after the media reports. 
A further analysis investigated whether the reports to CARM of the specific side effects mentioned in the February New Zealand Herald article increased in March and May compared to the five previous months. Figure 2 shows the rate of reporting for the mediamentioned side effects and Table 1 shows the interruption effects and corresponding $p$ values.

\section{Side Effects Mentioned in Article}

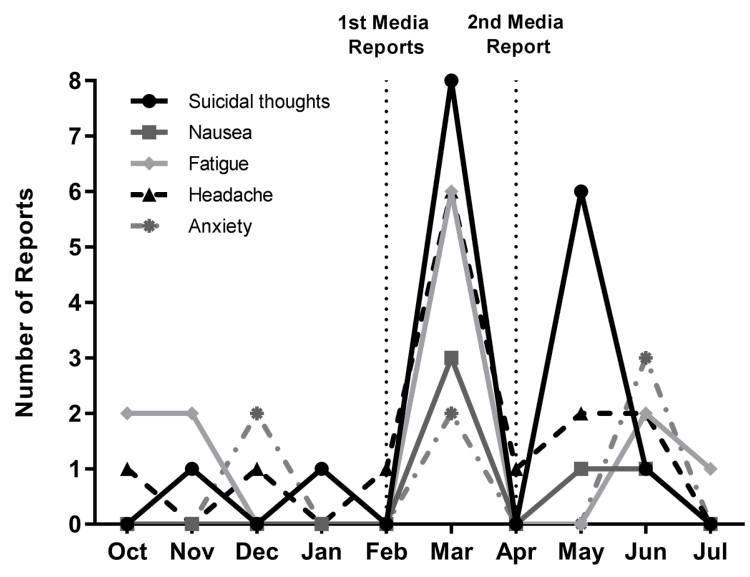

Side Effects Not Mentioned in Article

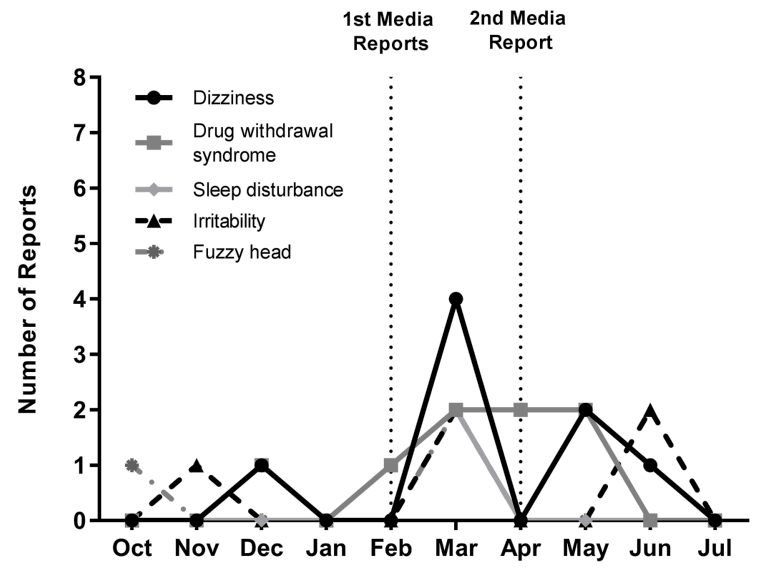

Figure 2. Numbers before and after media reports for the specific side effects reported in the media and control symptoms not in media reports. 
Table 1

Estimated Interruption Effects of the Newspaper Articles on CARM Reports for Specifically Mentioned Side Effects and Control Side Effects

\begin{tabular}{lccr}
\hline Variable & Interruption effect & $\mathbf{9 5 \%} \mathbf{C I}$ & $\boldsymbol{p}$ \\
\hline Side effects mentioned in article & & & \\
$\quad$ Suicidal thoughts & 6.64 & {$[4.60,8.68]$} & $<.001$ \\
$\quad$ Nausea & 1.95 & {$[0.62,3.28]$} & .029 \\
Fatigue & 1.63 & {$[-1.45,4.71]$} & .339 \\
Headache & 3.62 & {$[1.05,6.19]$} & .034 \\
Anxiety & 0.39 & {$[-2.34,3.11]$} & .791 \\
Side effects not mentioned in article & & & \\
Dizziness & 2.70 & {$[1.72,4.60]$} & .002 \\
Drug withdrawal syndrome & 2.96 & {$[0.53,5.39]$} & .055 \\
Sleep disturbance & 0.75 & {$[0.20,1.30]$} & .036 \\
Irritability & 0.50 & {$[-0.91,1.91]$} & .507 \\
$\quad$ Fuzzy head & 0.88 & {$[-0.30,2.06]$} & .190 \\
\hline
\end{tabular}

Prior to the media coverage, suicidal thoughts were reported an average of 0.40 times $(S D=0.55)$ per month but following the media report this significantly increased to 7.00 $(S D=1.41)$ across March and May. There were no adverse event reports of nausea before the media coverage, but reporting significantly increased to $2.00(S D=1.41)$ during the post-media months. The average rate of reporting per month of headache was $0.60(S D=$ $0.55)$ before the media focus, which significantly increased to $4.00(S D=2.83)$ reports in March and May. Fatigue was reported 0.80 times $(S D=1.10)$ over the five pre-media months but this did not change significantly after the media coverage $(M=4.00, S D=$ 4.24). Similarly, the reporting of anxiety did not change, going from an average of 0.40 $(S D=0.89)$ before the media coverage to $1.00(S D=1.41)$ after the focus.

The side effects most frequently reported to CARM that were not mentioned in the newspaper article were investigated to determine the effect on other adverse events. Dizziness, sleep disturbance, irritability and fuzzy head were all reported an average of 0.20 times $(S D=0.45)$ per month before the media focus. Following the coverage, there was a significant increase in the reporting of dizziness $(M=3.00, S D=1.41)$ and sleep disturbance $(M=1.00, S D=1.41)$. There was no change in the post-media rate of reporting for irritability and fuzzy head (both $M=1.00, S D=1.41$ ). Before the media articles, drug withdrawal syndrome was reported an average of 0.40 times $(S D=0.55)$ a month, which did not change after the media coverage $(M=2.00, S D=0)$. 


\section{Discussion}

\section{Main Findings}

This study found that reports by the two largest New Zealand media outlets highlighting the side effects and lack of efficacy of a new generic antidepressant were followed by a significant increase in reports to CARM of similar side effects. The increase in reported adverse events was largely limited to those mentioned in the media reports. While two of the control symptoms, dizziness and sleep disturbance, did also increase, this was at a lower rate than the symptoms mentioned in the media stories. The results are consistent with a nocebo response driven by the media coverage, whereby patients' expectations of particular side effects result in an increase in those specific symptoms. A pharmacological explanation for this effect is very unlikely as the side effects highlighted in the media stories and the control side effects were mentioned at a similar rate prior to the media coverage. Following the media coverage, it was those symptoms mentioned in the media stories that were mostly affected.

A particular concern in the findings is the mirroring of reports of decreased therapeutic efficacy, which could potentially drive non-persistence with antidepressant therapy. Also of public health relevance is the increase in reports of suicidal ideation which is likely due to the highlighting of suicidal thoughts and behaviour by patients discussed in the media stories in February and again in April.

\section{Comparison With Other Studies}

Previous studies have shown that information about likely side effects from medication can result in a significant increase in reports of those specific effects. Patients who were told about sexual side effects when starting finasteride or beta-blocker medication were significantly more likely to report these symptoms than patients who were not told of these side effects (Cocco, 2009; Mondaini et al., 2007). Similarly, in the context of a clinical trial, those patients warned of gastrointestinal side effects in one research site were more likely to complain of this as a side effect and withdraw from the study due to these complaints (Myers, Cairns, \& Singer, 1987).

Seeing another person in a media story report side effects from a medication can also increase the expectations of a similar response (Faasse \& Petrie, 2016). In an earlier study by our group, media reporting on a change in the formulation and appearance of thyroxine replacement therapy that led to a dramatic increase in adverse reaction reports (Faasse, Cundy, \& Petrie, 2009), found side effect complaints increased significantly after television news stories. The largest increases concerned symptoms mentioned in the media reports. This was strongest for the initial coverage and weakened with successive stories (Faasse, Gamble, Cundy, \& Petrie, 2012). This occurred in the current study, as the May adverse event reporting was not as large as in March. Research on side effects from 
electromagnetic fields has also shown that alarmist media reports, which emphasise adverse effects, exacerbate the nocebo effect and lead to greater symptom reporting and a perceived sensitivity to the supposedly harmful substance (Verrender, Loughran, Dalecki, Freudenstein, \& Croft, 2018; Witthöft \& Rubin, 2013).

Switches to generic medicine provide potential for a nocebo response to be strongly influenced by negative media coverage as non-adherence, patient reports of decreased efficacy and increased side effects are more common following switches (Boone et al., 2018; Weissenfeld, Stock, Lüngen, \& Gerber, 2010). A nocebo response induced through media reports can have a detrimental effect. Recent work has shown that negative stories in the media about statins have led to an increase in the rate of patients discontinuing statins in the United Kingdom (Matthews et al., 2016) and this early discontinuation has been linked to an increase in myocardial infarction and death from cardiovascular disease in Denmark (Nielsen \& Nordestgaard, 2016).

The likely mechanisms of the nocebo response found in this study are social transmission and the misattribution of common symptoms to the effects of the new medication (Petrie \& Rief, 2019). Previous research has found that seeing another person report side effects after receiving a treatment increases the likelihood of side effects' complaints after receiving the same treatment, especially if the observer can empathise with the person reporting the side effects (Faasse, Parkes, Kearney, \& Petrie, 2018). Studies have also found that individuals with higher levels of psychological distress also report a greater number of physical symptoms (Watson \& Pennebaker, 1989). This is likely to be of more relevance in this group of patients taking an antidepressant and thus allowing more symptoms to be misattributed to the effects of the new generic medicine. While generic switches are now commonplace in New Zealand, a recent general population survey found 38\% still preferred taking branded medication compared to a generic or no preference (Kleinstäuber, MacKrill, \& Petrie, 2018). Following a switch to a generic medicine more side effects are reported by patients who are older, female and by those who have been on their previous branded medicine longer (MacKrill \& Petrie, 2018).

\section{Strengths and Limitations}

While this study drew on adverse reports to a national database, it is likely that the rates are a substantively low estimate of the true effect of the nocebo effect caused by the media coverage. Studies estimate that reports to a national adverse database represent less than $10 \%$ of detected adverse drug reactions (McGettigan, Golden, Conroy, Arthur, \& Feely, 1997; Smith et al., 1996). It is further likely that many patients would not have sought medical assistance for symptoms due to the perception that there was little that could be done by their GP.

The study is limited by the non-experimental design and restricted in outcomes to the specific side effect categories recorded by CARM. As such, it is possible that patients may have experienced other side effects that the CARM database does not measure. Although 
patients can make direct reports using online forms this makes up only a small percentage of CARM reports. The behavioural outcomes of the adverse event reporting are also unknown. It is not known whether there was an increase in suicidal behaviour following the stories or whether patients stopped venlafaxine or changed to another medication.

In conclusion, we found media coverage of reports of a lack of efficacy and side effects following a switch to a generic version of venlafaxine were likely responsible for an increase in similar reports to a national centre for adverse drug reactions. Of particular concern is how media reports of increases in suicidal thoughts and loss of drug efficacy following a drug switch can be readily converted in similar complaints across the wider community. More research is also required on how such media reports are associated with increases in non-adherence and non-persistence with medication, as well as possible increases in suicidal behaviour. Future work may also be needed to develop guidelines for media reporting on generic switches with a view to avoiding these adverse outcomes.

Funding: The authors have no funding to report.

Competing Interests: KM, GG, DB, TC declare no conflicts of interest. KP has received research grants in the past from Pharmac, the New Zealand Government's Pharmaceutical Management Agency.

Acknowledgments: The authors have no support to report.

\section{References}

Boone, N. W., Liu, L., Romberg-Camps, M. J., Duijsens, L., Houwen, C., van der Kuy, P. H. M., . . van Bodegraven, A. A. (2018). The nocebo effect challenges the non-medical infliximab switch in practice. European fournal of Clinical Pharmacology, 74(5), 655-661.

https://doi.org/10.1007/s00228-018-2418-4

Cocco, G. (2009). Erectile dysfunction after therapy with metoprolol: The hawthorne effect. Cardiology, 112(3), 174-177. https://doi.org/10.1159/000147951

Colgan, S., Faasse, K., Martin, L. R., Stephens, M. H., Grey, A., \& Petrie, K. J. (2015). Perceptions of generic medication in the general population, doctors and pharmacists: A systematic review. BMF Open, 5(12), Article e008915. https://doi.org/10.1136/bmjopen-2015-008915

Desmarais, J. E., Beauclair, L., \& Margolese, H. C. (2011). Switching from brand-name to generic psychotropic medications: A literature review. CNS Neuroscience \& Therapeutics, 17(6), 750-760. https://doi.org/10.1111/j.1755-5949.2010.00210.x

Faasse, K., Cundy, T., Gamble, G., \& Petrie, K. J. (2013). The effect of an apparent change to a branded or generic medication on drug effectiveness and side effects. Psychosomatic Medicine, 75(1), 90-96. https://doi.org/10.1097/PSY.0b013e3182738826

Faasse, K., Cundy, T., \& Petrie, K. J. (2009). Thyroxine: Anatomy of a health scare. BMf: Clinical Research Edition, 339, Article b5613. https://doi.org/10.1136/bmj.b5613 
Faasse, K., Gamble, G., Cundy, T., \& Petrie, K. J. (2012). Impact of television coverage on the number and type of symptoms reported during a health scare: A retrospective pre-post observational study. BMF Open, 2(4), Article e001607. https://doi.org/10.1136/bmjopen-2012-001607

Faasse, K., Martin, L. R., Grey, A., Gamble, G., \& Petrie, K. J. (2016). Impact of brand or generic labeling on medication effectiveness and side effects. Health Psychology, 35(2), 187-190. https://doi.org/10.1037/hea0000282

Faasse, K., Parkes, B., Kearney, J., \& Petrie, K. J. (2018). The influence of social modeling, gender and empathy on side effects. Annals of Behavioral Medicine, 52(7), 560-570. https://doi.org/10.1093/abm/kax025

Faasse, K., \& Petrie, K. J. (2013). The nocebo effect: Patient expectations and medication side effects. Postgraduate Medical fournal, 89(1055), 540-546. https://doi.org/10.1136/postgradmedj-2012-131730

Faasse, K., \& Petrie, K. J. (2016). From me to you: The effect of social modeling on treatment outcomes. Current Directions in Psychological Science, 25(6), 438-443. https://doi.org/10.1177/0963721416657316

Henry, D. (2018, February 28). Patients say generic Pharmac-funded version of antidepressant venlafaxine left them depressed, anxious. The New Zealand Herald. Retrieved from http://www.nzherald.co.nz

Kleinstäuber, M., MacKrill, K., \& Petrie, K. J. (2018). Characteristics of individuals who prefer branded innovator over generic medicines: A New Zealand general population survey. Drugs \& Therapy Perspectives, 34(10), 478-483. https://doi.org/10.1007/s40267-018-0541-z

Leclerc, J., Blais, C., Rochette, L., Hamel, D., Guénette, L., \& Poirier, P. (2017). Impact of the commercialization of three generic angiotensin II receptor blockers on adverse events in Quebec, Canada: A population-based time series analysis. Circulation: Cardiovascular Quality and Outcomes, 10(10), Article e003891. https://doi.org/10.1161/CIRCOUTCOMES.117.003891

MacKrill, K., \& Petrie, K. J. (2018). What is associated with increased side effects and lower perceived efficacy following switching to a generic medicine? A New Zealand cross-sectional patient survey. BMJ Open, 8(10), Article e023667. https://doi.org/10.1136/bmjopen-2018-023667

Matthews, A., Herrett, E., Gasparrini, A., Van Staa, T., Goldacre, B., Smeeth, L., \& Bhaskaran, K. (2016). Impact of statin related media coverage on use of statins: Interrupted time series analysis with UK primary care data. BMF: Clinical Research Edition, 353, Article i3283. https://doi.org/10.1136/bmj.i3283

Maude, S. (2018, February 28). Anti-depressant swap: Sufferers claim generic drug is harming their condition. Stuff. Retrieved from https://www.stuff.co.nz

McGettigan, P., Golden, J., Conroy, R. M., Arthur, N., \& Feely, J. (1997). Reporting of adverse drug reactions by hospital doctors and the response to intervention. British fournal of Clinical Pharmacology, 44(1), 98-100. https://doi.org/10.1046/j.1365-2125.1997.00616.x

Mondaini, N., Gontero, P., Giubilei, G., Lombardi, G., Cai, T., Gavazzi, A., \& Bartoletti, R. (2007). Finasteride $5 \mathrm{mg}$ and sexual side effects: How many of these are related to a nocebo 
phenomenon? Journal of Sexual Medicine, 4(6), 1708-1712.

https://doi.org/10.1111/j.1743-6109.2007.00563.x

Myers, M. G., Cairns, J. A., \& Singer, J. (1987). The consent form as a possible cause of side effects. Clinical Pharmacology and Therapeutics, 42(3), 250-253. https://doi.org/10.1038/clpt.1987.142

Nielsen, S. F., \& Nordestgaard, B. G. (2016). Negative statin-related news stories decrease statin persistence and increase myocardial infarction and cardiovascular mortality: A nationwide prospective cohort study. European Heart fournal, 37(11), 908-916.

https://doi.org/10.1093/eurheartj/ehv641

Petrie, K. J., \& Rief, W. (2019). Psychobiological mechanisms of placebo and nocebo effects: Pathways to improve treatments and reduce side effects. Annual Review of Psychology, 70(1), 599-625. https://doi.org/10.1146/annurev-psych-010418-102907

Smith, C. C., Bennett, P. M., Pearce, H. M., Harrison, P. I., Reynolds, D. J. M., Aronson, J. K., \& Grahame-Smith, D. G. (1996). Adverse drug reactions in a hospital general medical unit meriting notification to the Committee on Safety of Medicines. British Fournal of Clinical Pharmacology, 42(4), 423-429. https://doi.org/10.1111/j.1365-2125.1996.tb00004.x

Steele, M. (2018). Fight over Pharmac's switch to generic anti-depressant brand continues. Stuff. Retrieved from https://www.stuff.co.nz

Verrender, A., Loughran, S. P., Dalecki, A., Freudenstein, F., \& Croft, R. J. (2018). Can explicit suggestions about the harmfulness of EMF exposure exacerbate a nocebo response in healthy controls? Environmental Research, 166, 409-417. https://doi.org/10.1016/j.envres.2018.06.032

Watson, D., \& Pennebaker, J. W. (1989). Health complaints, stress and distress: Exploring the central role of negative affectivity. Psychological Review, 96(2), 234-254.

https://doi.org/10.1037/0033-295X.96.2.234

Weissenfeld, J., Stock, S., Lüngen, M., \& Gerber, A. (2010). The nocebo effect: A reason for patients' non-adherence to generic substitution? Die Pharmazie, 65(7), 451-456.

Witthöft, M., \& Rubin, G. J. (2013). Are media warnings about the adverse health effects of modern life self-fulfilling? An experimental study on idiopathic environmental intolerance attributed to electromagnetic fields (IEI-EMF). Journal of Psychosomatic Research, 74(3), 206-212.

https://doi.org/10.1016/j.jpsychores.2012.12.002 